\title{
ARTICLE
}

\section{Energy and angular dependence of the small-type OSL dosimeter in nuclear medicine regions using Monte Carlo simulation}

\author{
Vergil Lorenzo E. Cruz ${ }^{\mathrm{a}^{*}}$, Tohru Okazaki ${ }^{\mathrm{a}}$, Hiroaki Hayashi ${ }^{\mathrm{b}, \mathrm{d}}$, Yoshiki Mihara ${ }^{\mathrm{b}}$, Takashi Asahara ${ }^{\mathrm{b}}$, \\ Natsumi Kimoto $^{\mathrm{b}}$, Hiroki Okino ${ }^{\mathrm{b}}$, Yuki Kanazawa ${ }^{\mathrm{b}}$, Takuya Hashizume ${ }^{\mathrm{a}, \mathrm{c}}$ and Ikuo Kobayashi ${ }^{\mathrm{a}}$ \\ ${ }^{a}$ Nagase Landauer, Ltd. Block C22-1, Suwa, Tsukuba, Ibaraki, Japan; ${ }^{b}$ Graduate School of Health Sciences, Tokushima University,

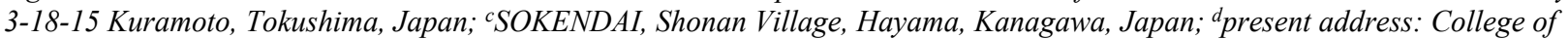 \\ Medical, Pharmaceutical and Health Sciences, Kanazawa University, 5-11-80 Kodatsuno, Kanazawa, Japan
}

Radiation doses received by patients and medical staff from examinations using X-rays and radioactive materials have been increasing, and a reliable radiation dosimetry system is a necessity. An OSL dosimeter can be used and applied in such situations. However at this time, its property for nuclear medicine region is unclear. When we calculate the basic properties in this region using the Monte-Carlo simulation code, the consideration of secondary electron equilibrium is important. The aim of this study is to propose a compact irradiation system for simulation study, and use it to evaluate the energy and angular dependence of the small-type OSL dosimeter. The proposed system uses a phantom of acrylic and we examined which phantom thickness will be used considering the accuracy of the simulation and the contamination from scattered rays. Then, this system was applied to determine angular and energy dependences of the small-type OSL dosimeter. Experiments using the OSL dosimeter were performed in order to check the accuracy of the simulations. In addition when simulating the response of the OSL dosimeter, we determined the basic parameters of the detection material. We performed several situations, and concluded that the following parameters were in good agreement with the experiment: a detection layer of $100 \% \mathrm{Al}_{2} \mathrm{O}_{3}$ with a density of $1.41 \mathrm{~g} / \mathrm{cm}^{3}$.

Keywords: OSL dosimeter; dosimetry system; Monte Carlo; EGS5; angular dependence; energy dependence; diagnostic region; nuclear medicine region

\section{Introduction}

A small-type optically stimulated luminescence (OSL) dosimeter has been used in medical dosimetry because of its good specifications. There have been many studies about characteristics of the OSL in the diagnostic X-ray and therapeutic region [1-8]. As well as low and high energy X-rays, various radioactive nuclei which emit gamma-rays are used in medical examinations, therefore, to expand the usable region of the OSL dosimeter in nuclear medicine, it is important to evaluate the response of the dosimeter for this region (up to $2500 \mathrm{keV}$ ). In this region, caused by various restrictions of half-lives and energies of $\gamma$-rays, ${ }^{137} \mathrm{Cs}$ and ${ }^{60} \mathrm{Co}$ are usually used. We considered that the simulation study is also valuable to evaluate the basic responses of the dosimeter.

Currently, Monte-Carlo simulation codes [9] can be used to mimic real experimental conditions, but it needs long simulation times when we want to obtain precise result. The long simulation times is needed especially for the nuclear medicine region because of the consideration of secondary electron equilibrium establishment. In this study, we examined the applicability of a compact irradiation system for simulating the detector response in the nuclear medicine region. The idea to establish the compact irradiation system is very simple; the OSL dosimeter was covered with an acrylic phantom having thickness of " $t$ ". At this time, the proper thickness and the accuracy of this irradiation (simulation) system were unclear. The objective of this study is to propose novel irradiation system for effective calculation of detector responses. Then, this system was applied to evaluate the energy and angular dependence of the small-type OSL dosimeter.

\section{Materials and method}

The Monte Carlo simulation code EGS5 [9] was used in this study. First, we proposed a compact irradiation system and evaluated the uncertainty of it. Next, we applied the system to evaluate the energy and angular dependence of the small-type OSL dosimeter called nanoDot (Nagase Landauer, Ltd., Japan). Then, experiments using nanoDots were performed to confirm the accuracy of the system.

*Corresponding author. vergil.cruz@nagase-landauer.co.jp 


\subsection{Compact irradiation system under consideration of secondary electron equilibrium}

Figure 1 shows the concept of irradiation system used in this study. For the diagnostic energy region $(0-200 \mathrm{keV})$, the secondary electron equilibrium can be established inside the detector; i.e. the cover of the detector plays as buildup cap. Therefore, it is adequate to set the irradiation field so as to cover full outer size of the nanoDot OSL dosimeter, which is $10 \mathrm{~mm}$ width, 10 $\mathrm{mm}$ length and $2 \mathrm{~mm}$ height, as shown in the left in Figure 1. On the other hand, for nuclear medicine region $(200-2500 \mathrm{keV})$, the additional phantom is needed to establish secondary electron equilibrium as shown in the right in Figure 1. Here, we defined the new irradiation system, which covers the nanoDot completely with the phantom thickness of " $t$ ". In this study, $t$ was chosen as 1,5 , and $10 \mathrm{~mm}$. In the irradiation system using three different phantom thicknesses, we examined the following two things; 1) whether secondary electron equilibrium is established, and 2) fraction of contamination from scattered rays.

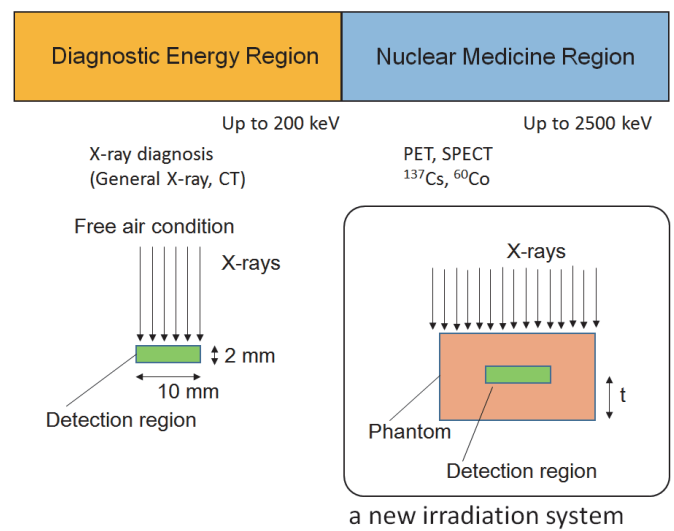

Figure 1. Schematic diagram of the concept of a compact irradiation condition. In the nuclear medicine region, a new irradiation system is applied in our simulation.

\subsection{Simulation of energy and angular dependences of nanoDot OSL dosimeter}

Using the compact irradiation system, we evaluated the angular and energy dependences of the nanoDot OSL dosimeter as follows. The nanoDot consists of the detection region and an outer plastic case. The dimensions of the outer case were described previously [5]. On the other hand, there were many different reports concerning the compositions and dimensions (thickness) for the detection region [8]. Additionally, only $\mathrm{Al}_{2} \mathrm{O}_{3}: \mathrm{C}$ can work to store the information corresponding to the absorbed dose though the actual detection parts were constructed with $\mathrm{Al}_{2} \mathrm{O}_{3}: \mathrm{C}$, polyester and glue. Thus, we adopted to use the unrealistic detection part in the present study; namely, the detection part consists of $100 \% \mathrm{Al}_{2} \mathrm{O}_{3}$ having an effective density of $1.41 \mathrm{~g} / \mathrm{cm}^{3}$ which was reported by a previous study as an experimental value of the actual dosimeter [6]. Under these assumptions, we calculated the energy and angular dependences of the nanoDot OSL dosimeter. The schematic diagram of the simulation is presented in Figure 2. For energy dependence evaluation from low energy photons, the nanoDot OSL dosimeter was irradiated from $10 \mathrm{keV}$ to $100 \mathrm{keV}$ at $10 \mathrm{keV}$ increments using the irradiation system of Figure. 2a). For angular dependence evaluation from low energy photons, the nanoDot OSL dosimeter was irradiated by X-rays assuming the tube voltage of $40 \mathrm{kV}$ from 0 to 360 degrees at 30 degrees increments using the irradiation system of Figure $2 \mathrm{c}$ ). For energy dependence evaluation from higher energy photons, energies of 100, 200, 300, $400,500,600,800,1000$ and $1500 \mathrm{keV}$ were irradiated to the OSL dosimeter surrounded by an acrylic phantom with thickness $t=5 \mathrm{~mm}$ as shown in Figure. $2 \mathrm{~b}$ ). For angular dependence evaluation from higher energy photons, energy of $1500 \mathrm{keV}$ were irradiated to the nanoDot OSL dosimeter surrounded by an acrylic with $t=5 \mathrm{~mm}$ from 0 to 360 degrees at 30 degrees increments using the irradiation system of Figure $2 \mathrm{~d}$ ). a)

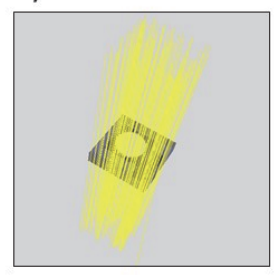

b)

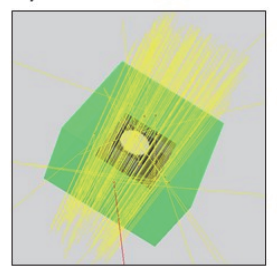

c)

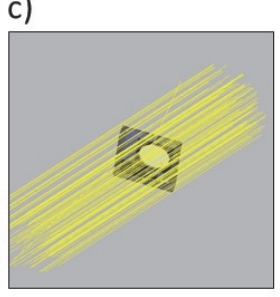

d)

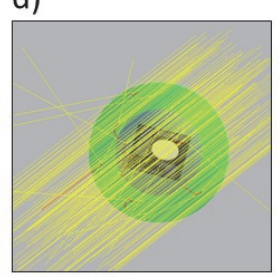

Figure 2. Three-dimensional views of simulated systems; a) energy dependence evaluation using free air condition for low energy photons, b) energy dependence evaluation using a phantom for higher energy photons, c) angular dependence evaluation for low energy photons, and d) angular dependence evaluation using phantom for higher energy photons.

\subsection{Experimental data of energy and angular dependence of nanoDot OSL dosimeter}

Experiments were also performed for energy dependence evaluation. Two types of radiation sources were used; one is X-ray equipment for medical diagnosis (laboratory standard) and the other is a Japanese primary standard constructed in the National Institute of Advanced Industrial Science and Technology (AIST) [10]. The tube voltages of medical diagnostic X-rays were between 40 and $140 \mathrm{kV}$ and mean energies of them were from 28 to $61 \mathrm{keV}$. The filtration of these X-ray spectra was $2.5 \mathrm{~mm}$ aluminum. For the aforementioned energies, 0.37-4.74 mGy were irradiated in free-air condition. The dosimeter inside of an acrylic phantom of which the thickness is $t=5 \mathrm{~mm}$ was irradiated with gamma-rays from a ${ }^{137} \mathrm{Cs}$ source with an energy of 662 $\mathrm{keV}$, and gamma-rays from a ${ }^{60} \mathrm{Co}$ source with a mean 
energy of $1253 \mathrm{keV}$ (cascade gamma-rays of $1332 \mathrm{keV}$ and $1173 \mathrm{keV}$ ) at a dose of $3.49 \mathrm{mGy}$.

\section{Results and discussion}

\subsection{Acrylic phantom and uncertainty evaluation}

The relationship between the incident photon energy and the range " $\mathrm{R}$ " of secondary electrons in the acrylic phantom was calculated by a well-known database [11]. An acrylic of $10 \mathrm{~mm}$ corresponds to a range $\mathrm{R}$ of an electron produced by photoelectric effect of $2320 \mathrm{keV}$ photons. However, secondary electron equilibrium is considered to be established in a smaller distance than $\mathrm{R}$ since electrons were scattered to various directions.

Table 1. Accuracy of simulation using our compact irradiation system.

\begin{tabular}{ccccc}
\hline \multirow{2}{*}{$\begin{array}{c}\text { Phantom } \\
\text { thickness } \\
{[\mathrm{mm}]}\end{array}$} & $\begin{array}{c}\text { Adoptable range } \\
{[\mathrm{keV}]}\end{array}$ & \multicolumn{3}{c}{ Uncertainty } \\
\cline { 3 - 5 } & & $\delta_{(\mathrm{D} / \mathrm{K})}$ & $\delta_{\text {sca }}$ & $\begin{array}{c}\text { Total: } \\
\delta_{\mathrm{t}}\end{array}$ \\
\hline 1 & $200-600$ & - & $1.1 \%$ & - \\
5 & $200-1500$ & $3.0 \%$ & $3.7 \%$ & $4.2 \%$ \\
10 & 200 -over 2500 & $3.0 \%$ & $5.2 \%$ & $5.6 \%$ \\
\hline
\end{tabular}

Figure 3 a) shows typical results of the relationship between the irradiation field size and the ratios of $\mathrm{D} / \mathrm{K}_{\mathrm{col}}$ where $\mathrm{D}$ and $\mathrm{K}_{\mathrm{col}}$ indicate absorbed dose and collision kerma corresponding to detection region. It is known that when secondary electron equilibrium is established, $\mathrm{D} / \mathrm{K}$ becomes 1.0 . In the case of $1500 \mathrm{keV}$ photons, it's clear that secondary electron equilibrium can be established even when the size of the irradiation field was set to $X^{\prime}=W+2 R$ ', where $W$ and $R^{\prime}$ is the mean length of the detection region and $\mathrm{R}^{\prime}=\mathrm{R} / 2$, respectively. It is well known that a sufficient irradiation field is established by considering range of secondary electron; in this case $\mathrm{X}=\mathrm{W}+2 \mathrm{R}$ is adequate for the secondary electron equilibrium. On the other hand, our result indicates that a smaller irradiation field with a side length of $\mathrm{X}$ ' can be practically used. The uncertainty caused by this procedure was evaluated by the deviation of $\mathrm{D} / \mathrm{K}$ values from the ideal value $(=1.0)$, also we determined the uncertainty as $\delta_{(\mathrm{D} / \mathrm{K})}=3.0 \%$. Figure $\left.3 \mathrm{~b}\right)$ shows calculation results of the fraction of scattered rays. The fraction rate depends strongly on the energy of photons. The maximum value in each thickness was derived, and we defined the value as an uncertainty caused by contamination of scattered rays. The corresponding uncertainty is defined as $\delta_{\text {sca }}$. After then, the total uncertainty of the system is evaluated by

$$
\delta_{t}=\sqrt{\left(\delta_{D / K}\right)^{2}+\left(\delta_{s c a}\right)^{2}}
$$

The uncertainties are summarized in Table 1. Based on these results, an acrylic phantom with thickness $t=5$ $\mathrm{mm}$ was considered to be proper to use for this study. (a) $D / K$

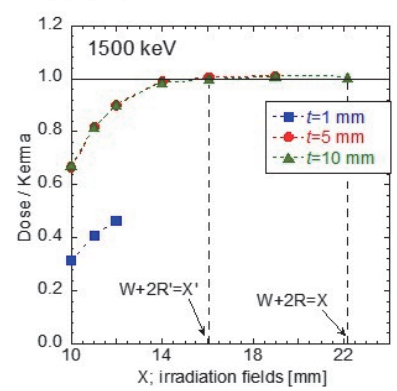

(b) Fraction of scattered rays

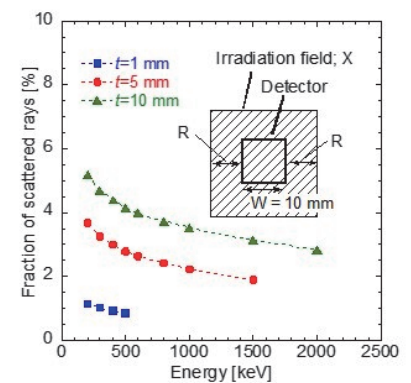

Figure 3. Typical results for simulation; (a) shows $\mathrm{D} / \mathrm{K}$ dependence as a function of size of irradiation fields. When using the proper phantom, the secondary electron equilibrium is established by $\mathrm{X}_{\text {eff. }}$ (b) shows fraction of scattered rays as a function of energy of photons.

\subsection{Energy Dependence}

Figure 4 shows a comparison of relative efficiencies between experiments and simulation. Here, efficiency is defined as a total energy deposition in the OSL dosimeter divided by the air-kerma of the irradiation filed, and the efficiency is normalized by that of ${ }^{60} \mathrm{Co}$. Although the present simulation is based on the unrealistic compositions in the detection region (100\% $\left.\mathrm{Al}_{2} \mathrm{O}_{3}, 1.41 \mathrm{~g} / \mathrm{cm}^{3}\right)$, the result was in good agreement with the experimental value. In this study, we carried out additional simulations, in which the effect of having different detector thicknesses of $0.15 \mathrm{~mm}$ and $0.20 \mathrm{~mm}$ on the relative efficiency. We found that different detector thicknesses made no significant differences in energy dependence, thus focus was given on $0.20 \mathrm{~mm}$. On the other hand, differences in the response due to detector material composition can be clearly observed. The relative efficiency of the $100 \% \mathrm{Al}_{2} \mathrm{O}_{3}$ is in good agreement with that of experiment, but relative efficiency of mixture of $\mathrm{Al}_{2} \mathrm{O}_{3}$ and polyester is much lower than that of the experiment. In addition, the differences in response due to the density of the material cannot be clearly defined.

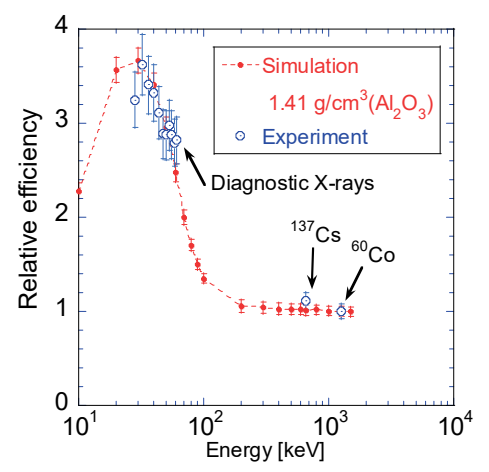

Figure 4. Energy dependence for the nanoDot OSL dosimeter.

\subsection{Angular dependence}

Figure 5 (a) shows a comparison of angular dependences of $40 \mathrm{kV}$ X-rays having $2.5 \mathrm{~mm}$ aluminum filtration between experiments and simulations. The experimental data from a previous study [5] of the 
angular dependences of the nanoDot OSL dosimeter was used in the present study. The simulated result is in good agreement with the experiments. Similar with the previous section, several conditions were also simulated to evaluate the accuracy of our simulation. The effect of having different detector thicknesses made no significant difference in angular dependence. Second, the composition of detection materials made no significant difference; result of $\mathrm{Al}_{2} \mathrm{O}_{3}$ indicates similar trend with that of composition materials of $\mathrm{Al}_{2} \mathrm{O}_{3}$ and polyester. Third, the angular dependences of 90 degrees and 270 degrees are sensitive with the density of detection material; the density of $1.41 \mathrm{~g} / \mathrm{cm}^{3}$ is the best result. Figure 5 (b) shows angular dependence evaluation for photon energy $E=1500 \mathrm{keV}$. Low efficiency was observed around 90 degrees and 270 degrees; the deviations were at most $10 \%$. The relative efficiency for angular dependence evaluation has better results at the middle energy region.
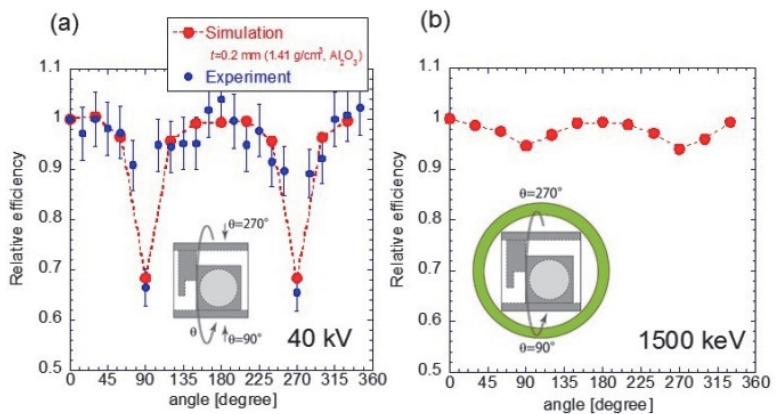

Figure 5. Angular dependences of the nanoDot OSL dosimeter for (a) $40 \mathrm{kV}$ X-rays and (b) $1500 \mathrm{keV}$ photons.

\subsection{Discussion}

The thickness of the detection layer does not affect the results significantly in the evaluation of the energy and angular dependence. On the other hand, precise description of the composition and the density of the detection layer are important in the simulation of the nanoDot OSL dosimeters. Results show that the composition of $100 \% \mathrm{Al}_{2} \mathrm{O}_{3}$ and the density of 1.41 $\mathrm{g} / \mathrm{cm}^{3}$ provide the best simulation results and were in good agreement with the experimental data. This result is something to think about since the density of $\mathrm{Al}_{2} \mathrm{O}_{3}$ was known to be $3.96 \mathrm{~g} / \mathrm{cm}^{3}$ [1]. Moreover, the effective density of the composition mixture of the $\mathrm{Al}_{2} \mathrm{O}_{3}$ and polyester is determined to be $1.41 \mathrm{~g} / \mathrm{cm}^{3}$ [6]. The non-realistic situation that the composition of only $\mathrm{Al}_{2} \mathrm{O}_{3}$ with a density of $1.41 \mathrm{~g} / \mathrm{cm}^{3}$ is available only in the simulation.

\section{Conclusion}

In this study, we proposed compact irradiation system which can establish the secondary electron equilibrium; the dosimeter was fully covered with a phantom having $5 \mathrm{~mm}$ thickness. The accuracy is estimated to be $4.2 \%$ by considering the uncertainty of simulation and contamination of scattering rays. Using this system, we determined the basic properties of the small-type OSL dosimeter; nanoDot. Although nanoDot has complex detection material, we can find a good parameter to reproduce experimental results.

The results of this study will be useful when using the nanoDot OSL dosimeter in managing and measuring the radiation dose received by patients and the medical staff and provides us with a better understanding of the characteristics of the OSL dosimeter.

\section{References}

[1] J.R. Kerns, S.F. Kry, N. Sahoo, D.S. Followill and G.S. Ibbott, Angular dependence of the nanoDot OSL dosimeter, Medical Physics 38 (2011), pp. 3955-3962. DOI 10.1118/1.3596533

[2] P.A. Jursinic, Characterization of optically stimulated luminescence dosimeters, OSLDs, for clinical dosimetric measurements, Medical Physics 34 (2007), pp. 4594-4604.

[3] K. Takegami, H. Hayashi, H. Okino, N. Kimoto, I. Maehata, Y. Kanazawa, T. Okazaki and I. Kobayashi, Practical calibration curve of small-type optically stimulated luminescence (OSL) dosimeter for evaluation of entrance skin dose in the diagnostic X-ray region, Radiological Physics and Technology 8 (2015), pp. 286-294.

[4] P.A. Jursinic, Angular dependence of dose sensitivity of nanoDot optically stimulated luminescent dosimeters in different radiation geometries, Medical Physics 42 (2015), pp. 5633-5641.

[5] H. Hayashi, K. Takegami, H. Okino, K. Nakagawa, T. Okazaki and I. Kobayashi, Procedure to measure angular dependences of personal dosimeters by means of diagnostic X-ray equipment, Medical Imaging and Information Sciences 32 (2015), pp.8-14.

[6] J. Lehmann, L. Dunn, J.E. Lye, J.W. Kenny, A.D.C. Alves, A. Cole, A. Asena, T. Kron and I.M. Williams, Angular dependence of the response of the nanoDot OSLD system for measurements at depth in clinical megavoltage beams, Medical Physics 41 (2014), pp. 061712-1-9.

[7] K. Takegami, H. Hayashi, H. Okino, N. Kimoto, I. Maehata, Y. Kanazawa, T. Okazaki, T. Hashizume and I. Kobayashi, Energy dependence measurement of small-type optically stimulated luminescence (OSL) dosimeter by means of characteristic X-rays induced with general diagnostic X-ray equipment, Radiological Physics and Technology 9 (2016), pp. 99-108. DOI 10.1007/s12194-015-0339-9

[8] H. Hayashi, Y. Mihara, Y. Kanazawa, E. Tomita, S. Goto, K. Takegami, T. Okazaki, T. Hashizume and VLE. Cruz, Necessity of direct dose measurement during current X-ray diagnosis, Medical Research Archives 5 (2017), pp. 1-20.

[9] H. Hirayama, Y. Namito, A.F. Bielajew, S.J. Wilderman and W.R. Nelson, The EGS5 Code 
System, SLAC Report number: SLAC-R-730, KEK Report number: 2005-8 (2007), pp. 1-441.

[10]ISO 4037-1:1996, $X$ and gamma reference radiation for calibrating dosemeters and doserate meter and for determining their response as a function of photon energy - Part1: Radiation characteristics and production methods.
[11]L. Pages, E. Bertel, H. Joffre and L. Sklavenitis, Energy loss, range, and bremsstrahlung yield for $10-\mathrm{KeV}$ to $100-\mathrm{MeV}$ electrons in various elements and chemical compounds, Atomic Data 4 (1972), pp. 1-127. 